\title{
An Identified LPV Model for Mobile Robots Navigation with Audio Features
}

\author{
Manuel Manzanares, Yolanda Bolea, Antoni Grau \\ Automatic Control Dept, Technical University of Catalonia at Barcelona, Spain \\ \{manolo.manzanares;yolanda.bolea;antoni.grau\}@upc.edu
}

\begin{abstract}
Non-speech audio is becoming more attractive to be used as features to mobile robots navigation in industrial environments. In this paper authors present their advances in determining robot's position in indoor spaces using as sound sources industrial machines. A novel model is build to locate the robot under different spaces. An identification process is used to obtain the LPV model and it is validated using a real robot. Some uncertainties due to the robot motion and other factors have been taken into account when determining the robot's position and the obtained results demonstrate the validity of the model.
\end{abstract}

\section{INTRODUCTION}

Indoor robot localization is an important issue in the field of robotics. So far, usually for this purpose overall odometer, camera, infrared sensor, ultra sonic sensor, mechanical wave and laser are mainly used. Nowadays the role of acoustic perception in autonomous robots, intelligent buildings and industrial environments is increasingly important and in the literature there are different works [1] [2].

It is very interesting the use of audio sensors according on the application. In industrial environments this type of sensors offers its main advantages: they are cheaper than other type of sensors, they hold a reach greater than the ultrasound sensors and they can cover a large area of exploration (with low directivity), they are not sensitive in front of changing light conditions, like cameras. Although audio sensors present low resolution to detect obstacles this fact is not too much relevant in industrial environments.

In an industrial plant to establish the transmission characteristics of a sound between a stationary audio source and a microphone in closed environment there are different study models: 1) the beam theory applied to the propagation of the direct audio waves and reflected audio waves in the room [12]; 2) the development of a lumped parameters model similar to the model used to explain the propagation of the electromagnetic waves in the transmission lines [12] and the study of the solutions given by the wave equation [11]. Other authors propose a transfer function of a room, denoted RTF (Room Transfer Function) that carries out to industrial plant applied sound model [3][9][10]. In these works the complexity to achieve the RFTs is evident as well as the need of a high number of parameters to model the complete acoustic response for a specific frequency range, moreover to consider a real environment presents an added difficulty (absorption coefficients, objects in the room...).
In this work we study how to obtain a real plant RTF. Due that this RTF will be used by a mobile robot to navigate in an industrial plant, we have simplified the methodology and our goal is to determinate the $x-y$ coordinates of the robot. In such a case, the obtained RFT will not present a complete acoustic response, but will be powerful enough to determine the robot's position. In an authors' previous works, [7][8][13] the navigation system was presented. The work investigated the feasibility of using sound features in the space domain for robot localization (in x-y plane) as well as robot's orientation detection. We proposed a robust sound-based indoor robot's pose $(x, y, \theta)$ detection system utilizing two microphones. For this reason, here the navigation system will be skipped and the work is focused in obtaining a more general model for rooms through audio features. This model is LPV (linear parameters varying) because the parameters of the model varying along the robot's navigation. The model is found by identification of two significant experiments in a room and validated with a real robot.

\section{SOUND MODEL IN A CLOSED ROOM}

The acoustical response of a closed room (with rectangular shape), where the dependence with the pressure in a point respect to the defined $(x, y, z)$ position is represented by the following wave equation:

$$
L x \frac{\partial^{2} p}{\partial x^{2}}+L y \frac{\partial^{2} p}{\partial y^{2}}+L z \frac{\partial^{2} p}{\partial z^{2}}+k^{2} p=0
$$

$L x, L y$ and $L z$ denote the dimensions of the length, width and height of the room with ideally rigid walls where the waves are reflected without loss, (1) is rewritten as [6]:

$$
p(x, y, z)=p_{1}(x) p_{2}(y) p_{3}(z)
$$

when the evolution of the pressure according to the time is not taken into account.

Then (2) is replaced in (1), and three differential equations can be derived and it is the same for the boundary condition. For example, $p_{1}$ must satisfy the equation:

$$
\frac{d^{2} p_{1}}{d x^{2}}+k_{x}^{2} p_{1}=0
$$

With boundary conditions in $x=0$ and $x=L x$ :

$$
\frac{d p_{1}}{d x}=0
$$

$k_{x}, k_{y}$ and $k_{z}$ constants are related by the following expression:

$$
k_{x}^{2}+k_{y}^{2}+k_{z}^{2}=k^{2}
$$

Equation (3) has as general solution:

$$
p_{1}(x)=A_{1} \cos \left(k_{x} x\right)+B_{1} \sin \left(k_{x} x\right)
$$


Through (3) and limiting this solution to the boundary conditions, constants in (5) take the following values:

$$
k_{x}=\frac{n_{x} \pi}{L_{x}} ; \quad k_{y}=\frac{n_{y} \pi}{L_{y}} \quad y \quad k_{z}=\frac{n_{z} \pi}{L_{z}}
$$

being $n_{x}, n_{y}$ and $n_{z}$ positive integers. Replacing these values in (5) the wave equation eigenvalues are obtained:

$$
k_{n_{x} n_{y} n_{z}}=\pi\left[\left(\frac{n_{x}}{L_{x}}\right)^{2}+\left(\frac{n_{y}}{L_{y}}\right)^{2}+\left(\frac{n_{z}}{L_{z}}\right)^{2}\right]^{1 / 2}
$$

The eigenfunctions or normal modes associated with these eigenvalues are expressed by:

$$
\begin{aligned}
& p_{n_{x} n_{y} n_{z}}(x, y, z)=C_{1} \cdot \cos \left(\frac{n_{x} \pi x}{L_{x}}\right) \cdot \cos \left(\frac{n_{y} \pi y}{L_{y}}\right) \cdot \cos \left(\frac{n_{z} \pi z}{L_{z}}\right) \cdot e^{j \omega t} \\
& e^{j w t}=\cos (w t)-j \sin (w t)
\end{aligned}
$$

being $C_{1}$ an arbitrary constant and introducing the variation of pressure in function of the time by the factor $e^{j \omega t}$. This expression represents a three dimensional stationary waves space in the room. Eigenfrequencies corresponding to (6) eigenvalues can be expressed by:

$$
\begin{aligned}
& f_{n_{x} n_{y} n_{z}}=\frac{c}{2 \pi} k_{n_{x} n_{y} n_{z}}, f_{n_{x} n_{y} n_{z}}=\sqrt{f_{n x}{ }^{2}+f_{n y}{ }^{2}+f_{n z}{ }^{2}} \text { and } \\
& f_{n_{x} n_{y} n_{z}}=\sqrt{\left(\frac{n_{x} c}{2 L_{x}}\right)^{2}+\left(\frac{n_{y} c}{2 L_{y}}\right)^{2}+\left(\frac{n_{z} c}{2 L_{z}}\right)^{2}},
\end{aligned}
$$

where $c$ is the sound speed. Therefore, the acoustic response of any close room presents resonance frequencies (eigenfrequencies) where the response of a sound source emitted in the room at these frequencies is maximum. The eigenfrequencies depend on the geometry of the room and also depend on the materials reflection coefficients, among other factors.

In our case, the transform function $f_{\mathrm{T}}$ (that relates the distance between feature space coefficients of each signal vs source signal (see [13]) with the distance between the points $(\mathrm{x}, \mathrm{y})$ in the space domain) is represented by (7), considering that the pressure is the square of the amplitude of sound signals $\left(S_{j}\right)$ for an specific time. The solution of this equation is real because imaginary numbers are neglected, and represented as:

$$
S_{j}(x, y)=C_{2} \cdot \cos \left(\frac{n_{d_{x y}} \pi d_{x y}}{L_{d_{x y}}}\right)
$$

where $C_{2}$ is an arbitrary constant.

Microphones obtain the environmental sound and they are located at a constant height $\left(\mathrm{z}_{1}\right)$ respect the floor, and thus the factor:

$$
\cos \left(\frac{n_{z} \pi z_{1}}{L_{z}}\right)
$$

is constant and therefore, if temporal dependency pressure respect the time is not considered, (7) is:

$$
p_{n_{x} n_{y} n_{z}}(x, y)=C_{2} \cdot \cos \left(\frac{n_{x} \pi x}{L_{x}}\right) \cdot \cos \left(\frac{n_{y} \pi y}{L_{y}}\right)
$$

TABLE I

RESONANT FREQUENCIES IN EXPERIMENTS 1 AND 2

\begin{tabular}{|c|c|c|c|c|c|c|}
\hline $\mathrm{n}_{\mathrm{x}}$ & $\mathrm{n}_{\mathrm{y}}$ & $\mathrm{n}_{\mathrm{z}}$ & $\mathrm{f}_{\mathrm{nx}}$ & $\mathrm{f}_{\mathrm{ny}}$ & $\mathrm{f}_{\mathrm{nz}}$ & $\mathrm{f}(\mathrm{Hz})$ \\
\hline 1 & 0 & 0 & 16,4 & 0,0 & 0,0 & 16,37 \\
\hline 2 & 0 & 0 & 32,7 & 0,0 & 0,0 & 32,73 \\
\hline 0 & 1 & 0 & 0,0 & 34,2 & 0,0 & 34,16 \\
\hline 1 & 1 & 0 & 16,4 & 34,2 & 0,0 & 37,88 \\
\hline 0 & 0 & 1 & 0,0 & 0,0 & 43,1 & 43,13 \\
\hline 1 & 0 & 1 & 16,4 & 0,0 & 43,1 & 46,13 \\
\hline 2 & 1 & 0 & 32,7 & 34,2 & 0,0 & 47,31 \\
\hline 2 & 0 & 1 & 32,7 & 0,0 & 43,1 & 54,14 \\
\hline 0 & 1 & 1 & 0,0 & 34,2 & 43,1 & 55,01 \\
\hline 1 & 1 & 1 & 16,4 & 34,2 & 43,1 & 57,40 \\
\hline 2 & 1 & 1 & 32,7 & 34,2 & 43,1 & 64,02 \\
\hline 0 & 2 & 0 & 0,0 & 68,3 & 0,0 & 68,32 \\
\hline 1 & 2 & 0 & 16,4 & 68,3 & 0,0 & 70,25 \\
\hline 0 & 2 & 1 & 0,0 & 68,3 & 43,1 & 80,79 \\
\hline 1 & 2 & 1 & 16,4 & 68,3 & 43,1 & 82,43 \\
\hline 0 & 0 & 2 & 0,0 & 0,0 & 86,3 & 86,25 \\
\hline 1 & 0 & 2 & 16,4 & 0,0 & 86,3 & 87,79 \\
\hline 0 & 1 & 2 & 0,0 & 34,2 & 86,3 & 92,77 \\
\hline 1 & 1 & 2 & 16,4 & 34,2 & 86,3 & 94,20 \\
\hline 0 & 0 & 3 & 0,0 & 0,0 & 129,4 & 129,38 \\
\hline 1 & 0 & 3 & 16,4 & 0,0 & 129,4 & 130,41 \\
\hline
\end{tabular}

In our experiments, $L_{x}=10.54 \mathrm{~m}, L_{y}=5.05 \mathrm{~m}$ and $L_{z}=4 \mathrm{~m}$, considering a sound speed propagation of $345 \mathrm{~m} / \mathrm{s}$, the corresponding resonant frequencies to the first propagation modes that we obtain are indicated in Table I.

In Table I the resonant frequency corresponding to the propagation mode $(1,1,2)$ can be observed. This frequency is close to $100 \mathrm{~Hz}$, that we will select from the signal spectrum when the climatic chamber is used (experiment 1), and very close to $93.2 \mathrm{~Hz}$, the selected frequency for experiment 2, a PCB insulator as sound source.

When (11) is applied in the experiments rooms, for mode $(1,1,2)$, this equation indicate the acoustic pressure in the rooms depending on the $x-y$ robot's position, and this is:

$$
p_{n_{x} n_{y} n_{z}}(x, y)=C_{2} \cdot \cos \left(\frac{\pi x}{10,54}\right) \cdot \cos \left(\frac{\pi y}{5,05}\right)
$$

With these ideal conditions and for an ideal value for constant $C_{2}=2$, the theoretic acoustic response in the rooms for this absolute value of pressure, and for this propagation mode, can be seen in Fig. 1.

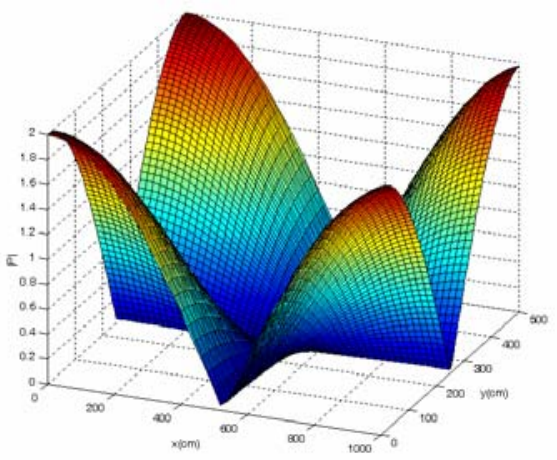

Fig. 1. Room response for propagation mode $(1,1,2)$. 
The shape of Fig. 1 would be obtained for a sound source that excited only this propagation mode, really the acoustic response will be more complex as we increase the propagation modes excited by the sound source.

\section{TRANSFER FUNCTION IN A CLOSED ROOM}

Since the dimensions of industrial plants will be comparable to the wavelength of audio signals presents in the environment, distributed constant models can be used in order to model the audio waves propagation, in a similar way to those proposed for electromagnetic signals transmission lines.

Even in closed rooms, as we stated before for other authors working with RTF, the number of parameters is relatively high for the description of RTF in our environment. Since the objective of this work is to find the $x-y$ coordinates through the processing of emitted audio signals for a fixed source, a new methodology is proposed in order to work without the need and constraint of a complete description of the plant's acoustic response.

In [4] a model based in the sum of second order transfer functions is proposed; these functions have been build between a sound source located in a position $d_{s}$ emitting an audio signal with a specific acoustic pressure $P_{s}$ and a microphone located in $d_{m}$ which receives a signal of pressure $P_{m}$; each function represents the system response in front to a propagation mode.

The first contribution of this work is to introduce an initial variation to this model considering that the sound source has a fixed location, then this model can be expressed as:

$$
\frac{P_{m}\left(d_{m}, s\right)}{P_{s}(s)}=\sum_{n=1}^{M} \frac{K\left[d_{m}\right] s}{s^{2}+2 \xi_{n} \omega_{n} s+\omega_{n}^{2}}
$$

Because our objective is not to obtain a complete model of the acoustic response of the industrial plant, it will not be necessary to consider all the propagation modes in the room and we will try to simplify the problem for this specific application without the need to work with models of higher order.

To implement this experiment the first step is to select the frequency of interest by a previous analysis of the audio signal frequency spectrum emitted by the considered sound source (an industrial machine). Those frequencial components with a significant acoustic power will be considered with the only requirement that they are close to one of the resonant frequencies of the environment. The way to select those frequencies will be through a band-pass digital filter centered in the frequency of interest. Right now, the term $M$ in the sum of our model will have the value $N$, being this new value the propagation modes resulting from the filtering process.

The spectra of the sound sources used in our experiments show an important component close to the frequency of $100 \mathrm{~Hz}$ for the climatic chamber, and a component of $50 \mathrm{~Hz}$ for the PCB insulator, see Fig. 3 (right) and Fig.4 (right).
IV.

For a concrete propagation mode, the variation that a stationary audio signal receives at different robot's position can be modeled, this signal can be smoothed by the variation of the absorption coefficient of the different materials that conform the objects in the room; those parameters are named $K\left[d_{m}\right]$ and $\xi\left[d_{m}\right]$, and (13) results:

$$
H\left(s, d_{m}\right)=\frac{P_{m}\left(d_{m}, s\right)}{P_{s}(s)}=\sum_{n=1}^{N} \frac{K\left[d_{m}\right] s}{s^{2}+2 \xi_{n}\left[d_{m}\right] \omega_{n} s+\omega_{n}^{2}}
$$

where the gain $(\mathrm{K})$, smooth coefficient $\left(\xi_{n}\right)$ and the natural frequency $\left(\omega_{n}\right)$ of the transfer function room system depend on the room characteristics: $d_{m}, n_{x}, n_{y}, L_{x}$, and $L_{y}$, yielding an LPV indoor model.

Using (13) the module of the closed room in a specific transmission mode $\omega_{n 1}$ is:

$$
\left|H\left(j \omega_{n 1}, d_{m 1}\right)\right|=\frac{K}{2 \xi_{n 1} \omega_{n 1}}
$$

The room response in the propagation mode $\omega_{n 1}\left(z_{1}\right.$ is a constant), assuming that the audio source only emits a frequency $\omega_{n 1}$ for a specific coordinate $(\mathrm{x}, \mathrm{y})$ of the room is:

$$
|H|=\left|\frac{P_{m}}{P_{s}}\right|_{n_{x}, n_{y}}=\left|C \cos \left(\frac{n_{x} \pi x}{L_{x}}\right) \cos \left(\frac{n_{y} \pi y}{L_{y}}\right)\right|
$$

with $f_{n_{1}}=\sqrt{f_{n x}{ }^{2}+f_{n y}^{2}}, \omega_{n_{1}}=2 \pi f_{n 1}$.

Equaling (15) and (16), it results:

$$
\xi_{n 1}=\frac{k}{2 \omega_{n 1}\left|\cos \left(\frac{n_{x} \pi x}{L_{x}}\right) \cos \left(\frac{n_{y} \pi y}{L_{y}}\right)\right|}
$$

If the filter is non ideal then more than one transmission mode could be considered and therefore the following expression is obtained:

$$
\sum_{l=1}^{m} \frac{K_{n l}}{2 \xi_{n l} \omega_{n l}}=\sum_{l=1}^{m} C\left|\cos \left(\frac{n_{x l} \tau x}{L_{x}}\right) \cos \left(\frac{n_{y l} \pi y}{L_{y}}\right)\right|
$$

The best results in the identification process in order to determine the robot's position have been obtained, for each considered propagation mode, keeping $K\left[d_{m}\right]$ coefficient constant and observing the different variations in the acquired audio signal in the smoothing coefficient $\xi\left[d_{m}\right]$.

If the zeros of the system are forced to be constant in the identification process for different robot's locations, and we admit that the emitted signal power by the sound sources are also constant and the audio signal power acquired with the microphones varies along the robot's position, then the pole positions in the $s$ plane, for the considered propagation mode, will vary in the different robot's positions and their values will be:

$$
\begin{aligned}
& s_{1 n}\left[d_{m}\right]=-\xi_{n}\left[d_{m}\right] \omega_{n}+\omega_{n} \sqrt{\left(\xi_{n}\left[d_{m}\right]\right)^{2}-1} \\
& s_{2 n}\left[d_{m}\right]=-\xi_{n}\left[d_{m}\right] \omega_{n}-\omega_{n} \sqrt{\left(\xi_{n}\left[d_{m}\right]\right)^{2}-1}
\end{aligned}
$$

It is worth noting that this model of reduced order gives good results in order to determine the robot's position and, although it does not provide a complete physical description of the evolution of the different parameters in the acoustic response for the different robot's positions, we can admit that 
according to the physical model given by the wave equation in (11), the modules of the proposed transfer functions will vary following a sinusoidal pattern and the pole position in the $s$ plane will show those variation in the same fashion.

\section{EXPERIMENTAL RESULTS}

The methodology applied to determine the robot's position is the following:

1) The robot acquires an audio signal in its current position and performs an identification process taking as input signal the filtered sound source signal and as output signal the acquired and filtered signal. The parameters corresponding to the obtained poles in this identification process will be the features components for further steps.

2) The Euclidean distances in the feature space are calculated between the current position and the different labeled samples.

3) The two first samples are chosen and the distance between them and the robot's position are then calculated. Through a transformation function $f_{T}$ (see [13]) the distance in the feature domain is converted to a distance in the space domain. These two distances in the space domain give two possible positions by the crossing circles of distances.

4) To discriminate between both possible solutions, the angle between each one and the platform containing the microphone array (which contains a compass) are calculated, and the closest one to the platform angle will be chosen as discriminatory variable to select the current robot's position.

5) Steps 3 and 4 are repeated with the remaining labeled samples, and the solution is chosen among the closest angle to the robot's platform.

For an accurate explanation of the algorithm, the microphone array and the robot used in the experiments see [13].

The acoustic response of the environment is very directional, and this fact leads to consider some uncertainty in the determination of the transformation function which relates the distance in the feature space and the domain space.

The robot, in order to determine its location, will perform the identification process between the emitted sound signal by the sound sources and the acquired signal by the microphone. Furthermore, the robot incorporates a rotary platform allowing orientating the microphone to the audio source, determining this orientation angle accurately.

As it can be seen in Fig. 2, the robot follows the trajectory indicated by the arrows. In the map the two sound sources are indicated (climate chamber and PCB insulator). Two experiments are carried out using both sound sources separately. There are two kind of audio samples: R1, R2, R3, R4, R5, R6 and R7 which are used in the recognition step whereas M1, M2, M3, M4 and M5 are labeled samples used in the learning step.

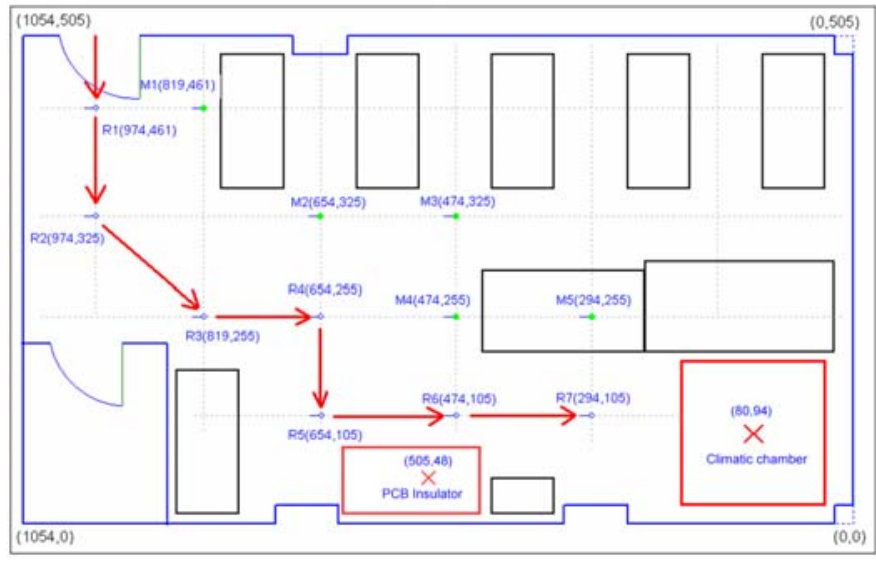

Fig. 2. Robot environment: labeled audio signals and actual robot trajectory with unlabeled signals (R1, R2, R3, R4, R5, R6 and R7).

The acquired signal in the climatic chamber will be used in the identification process. This signal is time-continuous and, initially, non-stationary; but because the signal is generated by revolving electrical machines it has some degree of stationariety when a high number of samples is used, in this case, 50,000 samples (1.13 seconds).
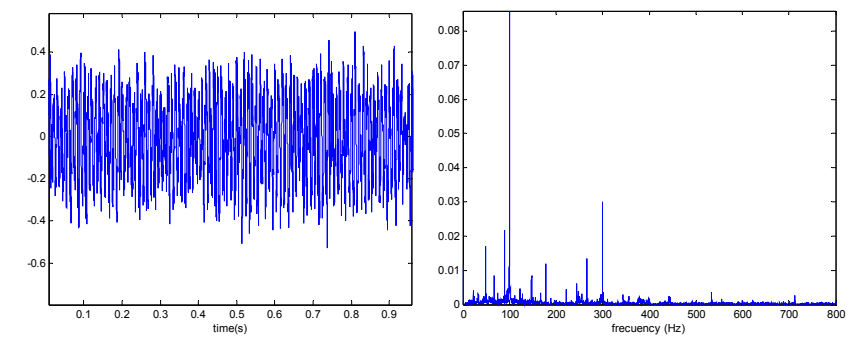

Fig. 3. Source signal (climate chamber) and its frequency spectrum.

The fundamental frequency is located at $100 \mathrm{~Hz}$, see Fig. 3, and there are also some significant harmonics above and below it. In order to simplify the identification process only the fundamental frequency at $100 \mathrm{~Hz}$ will be taken into account.

The sampling frequency is $44,100 \mathrm{~Hz}$. Other lower frequencies could be used instead, avoiding working with a high number of samples, but this frequency has been chosen because in a near future a voice recognition system will be implemented aboard the robot and it will be shared with this audio localization system.

The emitted signal for the PCB insulator machine and its spectrum can be seen in Fig 4 .
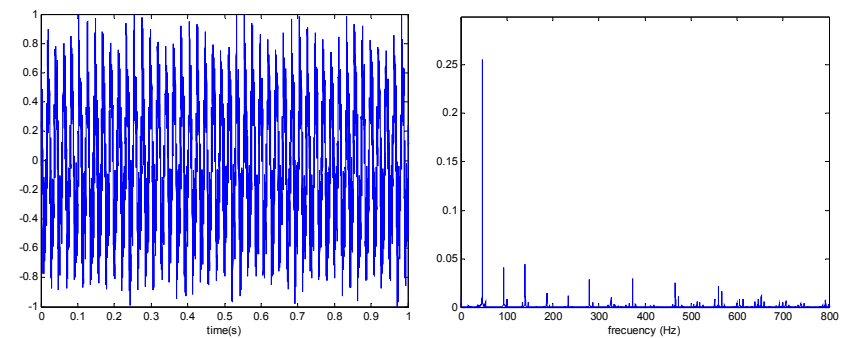

Fig.4. Source signal (PCB insulator)and its frequency spectrum. 
To facilitate the plant identification process centering its response in the $100 \mathrm{~Hz}$ component, the input and output signals will be filtered and, consequently, the input-output relationship in linear systems is an ARX model.

To do that, a band-pass filter is applied to the acquired sound signals by the robot, specifically a $6^{\text {th }}$-order digital Cauer filter. Fig. 5 shows the results of the filter for the input signal in, for instance, robot position $R_{4}$ in the climatic chamber (experiment 1).
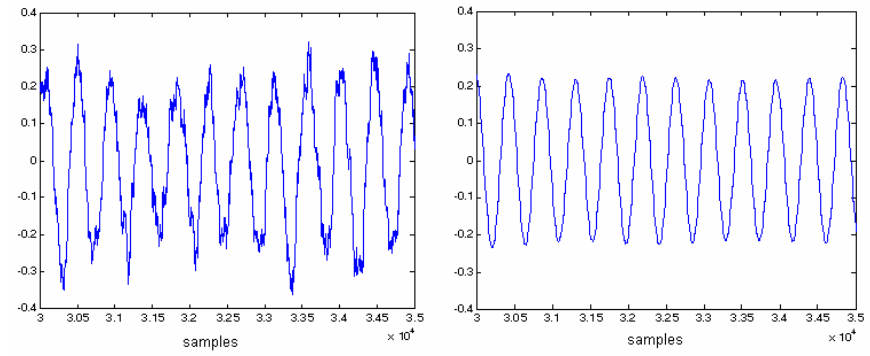

Fig. 5. R4 sound signal (left) and its filtered signal (right).

After an initial step for selecting the model structure, an ARX has been selected, for the reasons explained above of stationery [5], with $n_{a}=10, n_{b}=4$ and a delay of 2 for the case of the climatic chamber (experiment 1 ), and $n_{a}=10, n_{b}=$ 2 and a delay of 4 in the case of PCB insulator (experiment 2 ). When those 5 models are calibrated, they are validated with the error criteria of FPE (Function Prediction Error) and MSE (Mean Square Error), yielding values about $10^{-10}$ and $3 \%$ respectively using 5000 data for identification and 3000 for validation. Besides, for the whole estimated models the residuals autocorrelation and cross-correlation between the inputs and residuals are uncorrelated, indicating the goodness of the models.

For instance, for labeled $M_{5}$ sample the signal and its estimation can be seen Fig. 6 in the first experiment, validating the model.

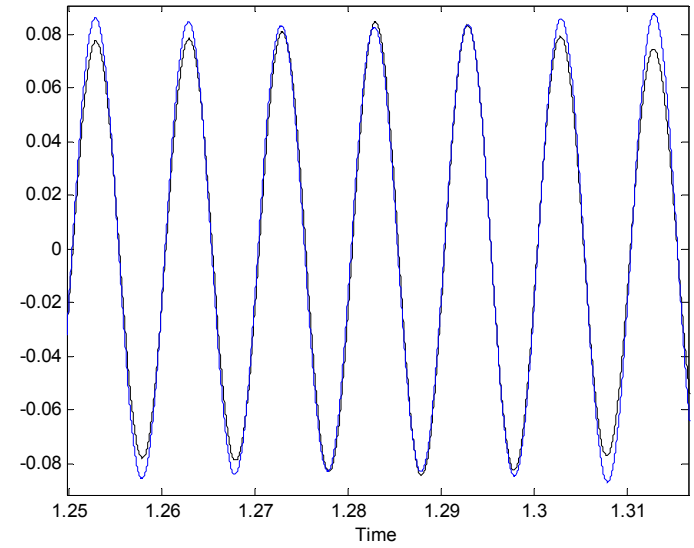

Fig. 6. Original M5 signal and its estimation.

When observing the diagram of poles and zeros for the different transfer function models in the identification process for the labeled signals, there exists no difference between the zero positions, and, in the other hand, there is a significant variation in pole positions, due mainly to obstacles presence, reverberations among other effects, see Fig. 7. Therefore, we will focus in poles to determinate the points in the feature space.
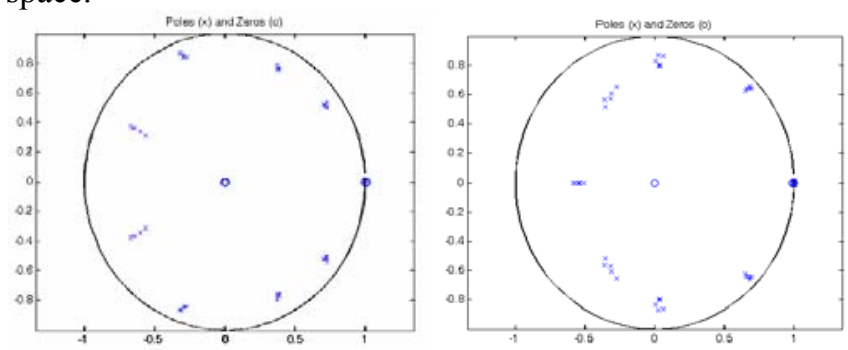

Fig. 7. Poles and zeros positions in experiment 1 (left) and 2 (right).

In experiment 1 , in order to determine the transformation function, for every point in the feature space, the distance between them and the source signal are calculated, and these distances are plotted together with their corresponding distances in the space domain. With these values, after an interpolation process, the transform function $f_{\mathrm{T}}$ is computed. In order to estimate the robot localization, we use other information such as the robot speed (in this case $15 \mathrm{~cm} / \mathrm{sec}$ ), the computation time between each new position $(3 \mathrm{sec})$. This fact is a source of uncertainty that adds in average $\pm 45 \mathrm{~cm}$ in the robot's position.

In experiment, when the climatic chamber is used as sound source the obtained transformation function is:

$$
y=4,4+4,4 \cdot \sin \left(\frac{2 \pi x}{170}-\frac{80 \pi}{170}\right)
$$

Now, if an uncertainty interval is supposed $( \pm 50 \mathrm{~cm})$ the transformation function that covers this variability in the robot's position can be expressed (for both experiments) as:

$$
y=A+A \cdot \sin \left(\frac{2 \pi x}{170 \pm 50}-\frac{\phi}{170 \pm 50}\right)
$$

In Fig. 8, the nominal transformation function and the limits for the uncertainty interval transformation functions can be seen.

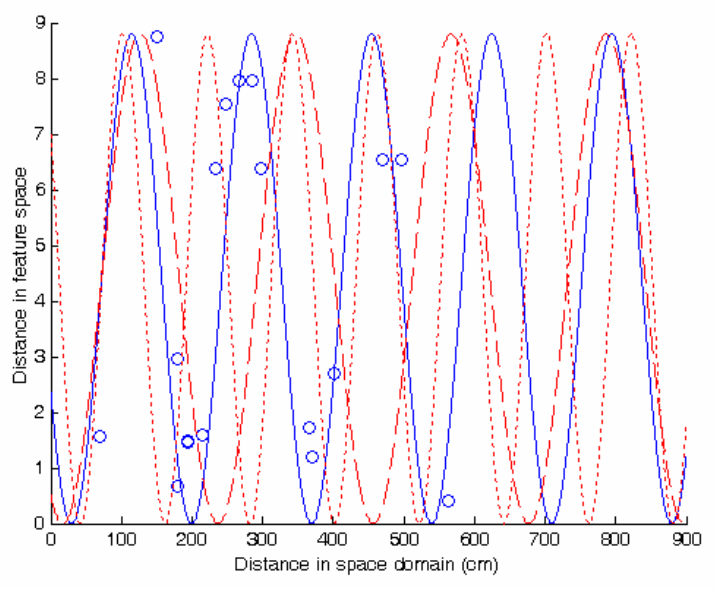

Fig. 8. Nominal transformation function and the limits of the interval for the uncertainty in experiment 1 . 
There exists another uncertainty of about \pm 7.5 degrees in the angle determination due to the rotary platform in the robot that contains the microphones. Finally, to determine the current robot's position the solution that provides the closest angle to the robot's platform will be chosen.

The results of our experiments are shown in Table II and Table III. The average error in the $\mathrm{X}$ axis is $-1.242 \%$ and in the $\mathrm{Y}$ axis is $0.454 \%$ in experiment 1 and $0.335 \%$ in the $\mathrm{X}$ axis and $-0.18 \%$ in the $\mathrm{Y}$ axis, providing estimated $\mathrm{x}-\mathrm{y}$ positions good enough and robust.

TABLE II

RESULTS FOR EXPERIMENT 1

\begin{tabular}{|c|c|c|c|c|}
\hline $\begin{array}{c}\text { Robot's } \\
\text { position }\end{array}$ & $\begin{array}{c}\text { Real } \\
\text { coordinates }\end{array}$ & $\begin{array}{c}\text { Computed } \\
\text { coordinates }\end{array}$ & $\begin{array}{c}\text { Error } \\
\mathrm{X}(\%)\end{array}$ & $\begin{array}{c}\text { Error } \\
\mathrm{Y}(\%)\end{array}$ \\
\hline R1(Pos1) & $(974,461)$ & $(973,456)$ & $-0,10$ & $-1,08$ \\
\hline R2(Pos2) & $(974,325)$ & $(978,321)$ & 0,41 & $-1,23$ \\
\hline R3(Pos6) & $(819,255)$ & $(807,250)$ & $-1,47$ & $-1,96$ \\
\hline R4(Pos10) & $(654,255)$ & $(644,257)$ & $-1,53$ & 0,78 \\
\hline R5(Pos11) & $(654,105)$ & $(640,110)$ & $-2,14$ & 4,76 \\
\hline R6(Pos15) & $(474,105)$ & $(475,108)$ & 0,21 & 2,86 \\
\hline R7(Pos19) & $(294,105)$ & $(282,104)$ & $-4,08$ & $-0,95$ \\
\hline
\end{tabular}

TABLE III

RESULTS FOR EXPERIMENT 2

\begin{tabular}{|c|c|c|c|c|}
\hline $\begin{array}{c}\text { Robot's } \\
\text { position }\end{array}$ & $\begin{array}{c}\text { Real } \\
\text { coordinates }\end{array}$ & $\begin{array}{c}\text { Computed } \\
\text { coordinates }\end{array}$ & $\begin{array}{c}\text { Error } \\
\mathrm{X}(\%)\end{array}$ & $\begin{array}{c}\text { Error } \\
\mathrm{Y}(\%)\end{array}$ \\
\hline R1(Pos1) & $(974,461)$ & $(976,449)$ & 0,21 & $-2,60$ \\
\hline R2(Pos2) & $(974,325)$ & $(990,331)$ & 1,64 & 1,85 \\
\hline R3(Pos6) & $(819,255)$ & $(805,247)$ & $-1,71$ & $-3,14$ \\
\hline R4(Pos10) & $(654,255)$ & $(642,269)$ & $-1,83$ & 5,49 \\
\hline R5(Pos11) & $(654,105)$ & $(650,100)$ & $-0,61$ & $-4,76$ \\
\hline R6(Pos15) & $(474,105)$ & $(488,102)$ & 2,95 & $-2,86$ \\
\hline R7(Pos19) & $(294,105)$ & $(299,110)$ & 1,70 & 4,76 \\
\hline
\end{tabular}

\section{CONCLUSIONS}

With the methodology presented in this article we have achieved some interesting results that encourage the authors to keep on walking in this research field. The room feature extraction is carried out by identification of the sound signals. Besides to reinforce the localization, avoiding ambiguity and reducing uncertainty and incorporating robustness, a sensorial system is used aboard the robot to compute the angle between itself and the sound source. The obtained feature space is related with the space domain through a general approach with acoustical meaning. The validation of this novel approach is tested in a room with two industrial machines as sound sources obtaining good results. The results keep being very good when the uncertainty is incorporated in the transformation function.

\section{ACKNOWLEDGMENT}

Authors thank the Inter-ministerial Commission of Spanish Government (DPI2004-5414, MCYT), the Generalitat of Catalonia (VIS, Consolidated Research Group) and the European Commission (FP6-2005-IST-6, URUS-045062) for funding the work.

\section{REFERENCES}

[1] E. Mumolo, M. Nolich, G. Vercelli, "Algorithms for acoustic localization based on microphone array in service robotics", Robotics and Autonomous Systems, vol. 42, pp.69-88, 2003.

[2] A. Csyzewski, "Automatic identification of sound source position employing neural networks and rough sets", Pattern Recognition Letters, vol.24, pp.921-933, 2003.

[3] Y. Haneda, S. Makino, Y. Kaneda, "Modeling of a Room Transfer Function Using Common Acoustical Poles", IEEE International Conference on Acoustics, Speech, and Signal Processing, ICASSP-92, pp. 213-216, vol.2 1992.

[4] L. Ljung, System identification: Theory for the user. Prentice-Hall, 1987.

[5] R. Charbonnier, M. Barlaud, G. Alengrin, J. Menez, "Results on ARmodeling of nonstationary signals", IEEE Trans. Signal Processing, vol 12, no. 2, pp. 143-151, 1987.

[6] A.S. Kayhan, A. Ei-Jaroudi, L.F. Chaparro, "Evolutionary periodogram for nonstationary signals", IEEE Trans. Signal Processing, vol. 42. no. 6, pp. 1527-1536, 1994.

[7] A. Grau, Y. Bolea, M. Manzanares, "Robust Industrial Machine Sounds Identification Based on Frequency Spectrum Analysis", 12th Iberoamerican Congress on Pattern Recognition, CIARP 2007, Lectures Notes in Computer Science, LNCS 4756, pp. 71-77, Santiago de Chile, 14-17 Novembre, 2007.

[8] Y. Bolea, M. Manzanares, A. Grau, "Robust robot localization using non-speech sound in industrial environments", IEEE International Symposium on Industrial Electronics, ISIE 2008, Cambridge, United Kingdom, 30 June- 2 July 2008.

[9] Y. Haneda, Y. Kaneda, N. Kitawaki, "Common-Acoustical-Pole and Residue Model and Its Application to Spatial Interpolation and Extrapolation of a Room Transfer Function", IEEE Transactions on Speech and Audio Processing, vol. 7, no. 6, November 1999.

[10] T. Gustaffson, H.R. Pota, J. Vance, B.D. Rao, M.M. Trivedi, "Estimation of Acoustical Room Transfer Functions", Proceedings of the 39th IEEE Conference on Decision and Control, Sydney, Australia, December 2000.

[11] H. Kuttruff, Room Acoustics, Applied Science Publishers Ltd. 1979.

[12] L. Kinsler, A. Frey, A. Coppens, J. Sanders, Fundamentals of Acoustics, Limusa Ed., Barcelona, 1995.

[13] M. Manzanares, E. Guerra, Y. Bolea, A. Grau "Robot Localization Method by Acoustical Signal Identification", IEEE Emerging Tech and Factory Automation, ETFA'09, Palma de Mallorca, Spain,. 2009. 